\title{
The Effect of Kyphosis Correction for Ankylosing Spondylitis by Hilus Pulmonis Approach
}

\author{
Li Shaojin, Luo Jianxian, Ji Zhisheng, Yang Yuhao, Zhang Guowei, Yang Hua, Lin Hongsheng* \\ Department of Orthopedics, The First Affiliated Hospital of Jinan University, Guangzhou, China
}

Email address:

tlinhsh@jnu.edu.cn (Lin Hongsheng)

${ }^{*}$ Corresponding author

\section{To cite this article:}

Li Shaojin, Luo Jianxian, Ji Zhisheng, Yang Yuhao, Zhang Guowei, Yang Hua, Lin Hongsheng. The Effect of Kyphosis Correction for Ankylosing Spondylitis by Hilus Pulmonis Approach. Clinical Medicine Research. Vol. 8, No. 6, 2019, pp. 131-136.

doi: $10.11648 /$ j.cmr.20190806.11

Received: October 16, 2019; Accepted: October 25, 2019; Published: October 30, 2019

\begin{abstract}
The efficacy of application of Hilus pulmonic in guiding of kyphosis correction was explored by radiographic parameters and the quality of life scores of AS kyphosis patients. A retrospective analysis was performed with patients that were diagnosed as ankylosing spondylitis with kyphosis and using the hilus pulmonic to guide kyphosis correction. A total of 16 cases, 15 males and 1 female, were enrolled. The spine-pelvic imaging parameter, the SRS-22 score and ODI dysfunction score were recorded and analyzed at pre-operation and post-operation. The post-operation spine-pelvic parameters PT, SS, GK, TLK, LL, SVA and TPA were all improved compared with preoperative parameters $(P<0.05)$. SRS-22 score and ODI score were significantly improved after osteotomy $(P<0.05)$. After using the Hilus pulmonic to guide kyphosis correction, patients with AS kyphosis can obtain good radiographic parameters and physical activity ability score, and can meet the functions of head-up, standing, sitting and lying in patients after orthopedic surgery. PT、SVA、GK and TPA have significant influences on the quality of life of patients with AS kyphosis. Correcting spine-pelvic imaging parameters is important guarantees of higher quality of life after surgery.
\end{abstract}

Keywords: Ankylosing Spondylitis, Kyphosis, Hilus Pulmonis Approach, Osteotomy Design

\section{Introduction}

Ankylosing spondylitis (AS) is a chronic inflammatory autoimmune disease that often begins with the involvement in the sacroiliac joints and gradually invades the entire spine. It also invades the attachment points, joints and other tissues of the peripheral tendon ligament [1]. Ankylosing spondylitis not only causes physical pain to the patient but also causes depression in the patient [2]. At present, the etiology and pathogenesis of AS have not been clarified and no effect treatment has been found in the clinic. Even in the early stage of well-preserved conservative treatment, in the late stage of the disease, $30 \%$ of AS patients have disappeared lumbar lordosis, resulting in kyphosis of thoracolumbar spine [3, 4]. Osteotomy and orthopedic surgery has become the only way to solve problems in advanced patients. With the understanding and research on pelvic rotation compensation and spine-pelvic parameters, Song proposed the approximation of the hilar as the image of the trunk center of gravity [5]. Learning mark, design guides osteotomy and orthopedic surgery, combined with the influence of cervical vertebrae activity and hip joint limitation to guide orthopedics. Postoperative hilar, humeral and hip axis ensure that the pelvis is in neutral position after orthopedic surgery.

In this study, the spine-pelvic imaging parameters and quality of life scores before and after orthopedic surgery in patients with AS kyphosis were evaluated to evaluate the short-term clinical efficacy of the hilar approach for guiding the treatment of osteotomy and the spine of patients with AS kyphosis. Through the correlation analysis between the patient's spine-pelvic parameters and the quality of life of patients, more reference is given to the design of osteotomy orthopedic surgery.

\section{Material and Methods}

\subsection{General Information}

Retrospective analysis was performed during the period from January 2014 to December 2017 at the Department of 
Orthopaedics, the First Affiliated Hospital of Jinan University. Paitients was diagnosed as ankylosing spondylitis with kyphosis and the use of the hilar as the center of the trunk to guide the posterior osteotomy. Orthopedic surgery for patients (16 samples) with complete follow-up data recorded clinical data (15 males and 1 female; the youngest is 20 years old, the oldest is 54 years old, average Age $36.12 \pm$ 8.80 years old).

\subsection{Inclusion Criteria}

(1) AS to meet the New York standard revised for 1984, with kyphosis parallel osteotomy;

(2) The kyphosis deformity is obvious, and no obvious effect is obtained after long-term conservative treatment;

(3) In the stationary phase of AS, the erythrocyte sedimentation rate is about $40 \mathrm{~mm}$;

(4) Follow-up for 12 months or more and complete data.

\subsection{Exclusion Criteria}

(1) Previous spine surgery;

(2) There is a pathological fracture or pseudoarthrosis;

(3) Defining spinal cord or nerve damage due to human manipulation factors;

(4) Other factors during the follow-up period have a clear impact on postoperative recovery and quality of life.

\subsection{Spinal Orthopedic Plan}

The overall orthopedic plan requires three parts: (1) Restoring the spine sequence: Calculate the theoretical PT by PI using the formula as $\mathrm{tPT}=0.37 \times \mathrm{PI}-7^{\circ}$ (tPT: theoretical pelvic tilt angle; PI: pelvic incident angle). The theoretical PT value, according to the lung door osteotomy orthopedic program, the hilar imaging mark as the trunk center of gravity, the hilar is reset on the tPT line, through the full length of the spine X On-chip measurements determine the osteotomy segment and the angle required to measure the osteotomy, and the osteotomy angle $\leq$ the measured angle. (2) Correction of the mandible eyebrow angle (CBVA): Patients with AS kyphosis can achieve good lower vision ability when obtaining the maxillary eyebrow angle of $10^{\circ} \sim 20^{\circ}$, and have satisfactory effects on housework activities and desk work. And design the osteotomy angle $\leq$ pre-operative jaw angle $-10^{\circ}+\Delta \mathrm{PT}(\Delta \mathrm{PT}$ is the change of preoperative pelvic tilt angle calculated by the hilar method). (3) Considering the influence of hip mobility: Considering the problem of pelvic rotation after osteotomy and orthopedics in patients with limited hip joint activity, a simple and easy osteotomy scheme was proposed.

\subsection{Surgical Methods}

After successful anesthesia, the patient was placed in a prone position, the abdomen was empty, the field was routinely disinfected, the towel was placed, and the sterile film was applied. According to the position of the osteotomy and the fixed range, the median longitudinal incision of the lower back is taken, and the skin, subcutaneous and deep fascia are sequentially cut, the paravertebral muscles are peeled off, and the bilateral articular processes are exposed until the root of the transverse process. Intraoperative positioning was performed to determine the osteotomy vertebral body, and the appropriate pedicle screw was placed according to the preoperative planning at the head and tail of the osteotomy vertebral body. In the osteotomy of the osteotomy of the osteotomy, the apex of the bilateral pedicle of the osteotomy vertebrae was treated with open circuit, ream and silk attack. Treatment of the bilateral pedicles of the osteotomy vertebral body from small to large using the osteotomy triangle allows the pedicle to rupture while maintaining the inner wall intact. The cancellous bone in the center of the vertebral body is treated by a truncated triangular pyramid to bring the endplates up and down. The dura mater is exposed by excising the posterior lamina of the osteotomy. The bilateral muscles of the bilateral vertebral bodies were dissected, and the vertebral bodies were cut with a bone knife and the cortical bone was removed. The nerve root stripper was used to free the dura mater, and the vertebral body osteotomy was used to treat the posterior cortical bone of the vertebral body and removed. The nerve root or the soft tissue around the spinal cord and the bone is treated, and the lower end of the vertebral body osteotomy is decompressed to avoid the compression during the resetting process. For double-segment osteotomy, the same procedure are performed for the osteotomy. Select a suitable length of the longitudinal connecting rod and pre-bend the connecting rod according to the osteotomy angle of the preoperative design. Insert one end of the rod into the pedicle screw tail, screw in the screw, slowly reset and adjust the position of the operating bed, and then put the connecting rod into the remaining screw tail groove. The intervertebral space at the osteotomy is appropriately symmetrically pressed in order, so that the osteotomy faces. Intraoperative fluoroscopy was performed to observe the osteotomy angle of the vertebral body and the position of the internal fixation. The incision was flushed without significant active bleeding. Indwell a wound drainage tube and suture the incision layer by layer. The entire surgical procedure was completed under neuroelectrophysiological monitoring.

\subsection{Evaluation Index: Measurement of Spinal Pelvis Parameters}

The patient stood a full-spine lateral image and measured the spine-pelvic imaging parameters before, during, and after 12 months of follow-up, including:

(1) Pelvicincidence (PI): the angle formed by the endplate of the tibia and the midpoint of the midpoint;

(2) Pelvictilt (PT): the angle formed by the central point of the hip shaft and the vertical line of the endplate of the humerus;

(3) Sacral slope (SS): the angle formed by the endplate of the humerus and the horizontal plane;

(4) Globe kyphosis (GK): T1 upper end plate connection for its vertical line and L5 vertebral lower end plate or S1 upper end plate connection for its vertical line, two vertical 
line clips Cobb angle between the corners, ie the full spine Cobb angle;

(5) Thoracolumbar kyphosis (TLK): the angle between the endplate of the T10 vertebral body and the lower endplate of the L2 vertebral body;

(6) lumbar lordosis (LL): the angle between the upper endplate of the L1 vertebral body and the upper endplate of the S1 vertebral body;

(7) Sagittal vertical axis (SVA): the horizontal distance between the $\mathrm{C} 7$ plumb line and the posterior superior anterior humerus;

(8) T1 pelvic angle (TPA): the angle formed between the midpoint of the endplate of the endplate and the midpoint of the femoral head on T1 and the midpoint of the femoral head and the midpoint of the upper endplate of S1.

The SRS-22 scores (higher scores suggest better patient function) and Oswestry disability index (ODI) were recorded before and after orthopedics for more than 12 months (the lower the score, the better the patient function). The activity ability scores of patients before and after orthopedics and the functional dyspepsia quality of life table were scored in Chinese. Among them, the activity ability ACS score was taken as the third major item in the SF-36 quality of life scale, "somatic activity function", a total of 10 questions, as the ACS score in this study, formula: ACS = (actual score - the minimum score) / (The maximum score - the minimum score), the higher the score, the better the physical activity.

\subsection{Statistical Analysis}

Statistical analysis was performed using SPSS19.0 statistical software. The imaging parameters and subjective function scores were compared before, after, and after 12 months of follow-up. $\mathrm{P}<0.05$ was considered statistically significant. Correlation analysis was performed between the imaging parameters before and after orthopedics and the quality of life scores. $\mathrm{P}<0.05$ showed a correlation and was statistically significant.

\section{Results}

\subsection{Spine-pelvic Parameters Were Analyzed at Pre-operation and Post-operation}

The paired t-test was used to analyze the pelvic parameters before and 2 weeks after surgery and 12 months after the operation. The PI values of the preoperative, 2 weeks and 12 months follow-up were $56.46 \pm 14.58^{\circ}$. 53.12 $\pm 13.39^{\circ}$ and $57.72 \pm 13.58^{\circ}$, no significant change, the difference was not statistically significant $(\mathrm{P}>0.05)$. The mean PTs before, 2, and 12 months after surgery were $37.89 \pm 4.65^{\circ}, 24.00 \pm 11.39^{\circ}$, and $25.98 \pm 9.65^{\circ}(\mathrm{P}<0.05)$, respectively. The mean SS of preoperative, postoperative 2 weeks and postoperative 12 months follow-up were $14.52 \pm 11.47^{\circ}, 28.70 \pm 9.10^{\circ}(\mathrm{P}<0.05)$ and $27.16 \pm 8.86^{\circ}(\mathrm{P}<0.05)$, respectively. This indicates that the pelvic posterior tilt state after the orthopedic surgery has been significantly improved. For the spine parameters before, after, and after 12 months of follow-up, the average GK of the preoperative, postoperative, and postoperative follow-up at 12 months was $42.70 \pm 24.74^{\circ}$ and $14.19 \pm 8.51^{\circ}$, respectively. $<0.05)$ and $14.57 \pm 9.48^{\circ}(\mathrm{P}<0.05)$, both showed significant improvement. The mean TLK of preoperative, postoperative 2 weeks and postoperative 12 months followup were $26.02 \pm 12.49^{\circ}, \quad 16.79 \pm 13.72^{\circ} \quad(\mathrm{P}<0.05)$ and $18.54 \pm 14.17^{\circ} \quad(\mathrm{P}<0.05)$, respectively. The mean LL of preoperative, postoperative 2 weeks and postoperative 12 months follow-up were $11.61 \pm 16.77^{\circ}, 43.23 \pm 15.80^{\circ}(\mathrm{P}<0.05)$ and $41.02 \pm 16.69^{\circ}(\mathrm{P}<0.05)$, respectively. The mean SVA of preoperative, postoperative 2 weeks and postoperative 12 months follow-up were $128.09 \pm 63.02 \mathrm{~mm}, 25.09 \pm 30.41 \mathrm{~mm}$ $(\mathrm{P}<0.05)$ and $34.48 \pm 23.07 \mathrm{~mm}(\mathrm{P}<0.05)$, respectively. The spine sequence was significantly repaired after orthopedic surgery. The mean TPA distribution of patients before, 2 weeks and 12 months after surgery was $42.78 \pm 18.73^{\circ}$, $23.57 \pm 11.25^{\circ}(\mathrm{P}<0.05), 27.23 \pm 9.91^{\circ}(\mathrm{P}<0.05)$, all of which showed significant improvement. There was a significant difference in the mean TPA between the 2 weeks postoperatively and the 12 -month follow-up period $(\mathrm{P}<0.05)$. There were no significant differences in the follow-up data of the remaining PI, PT, SS, GK, TLK, LL, SVA after 2 weeks and 12 months after surgery $(\mathrm{P}>0.05)$.
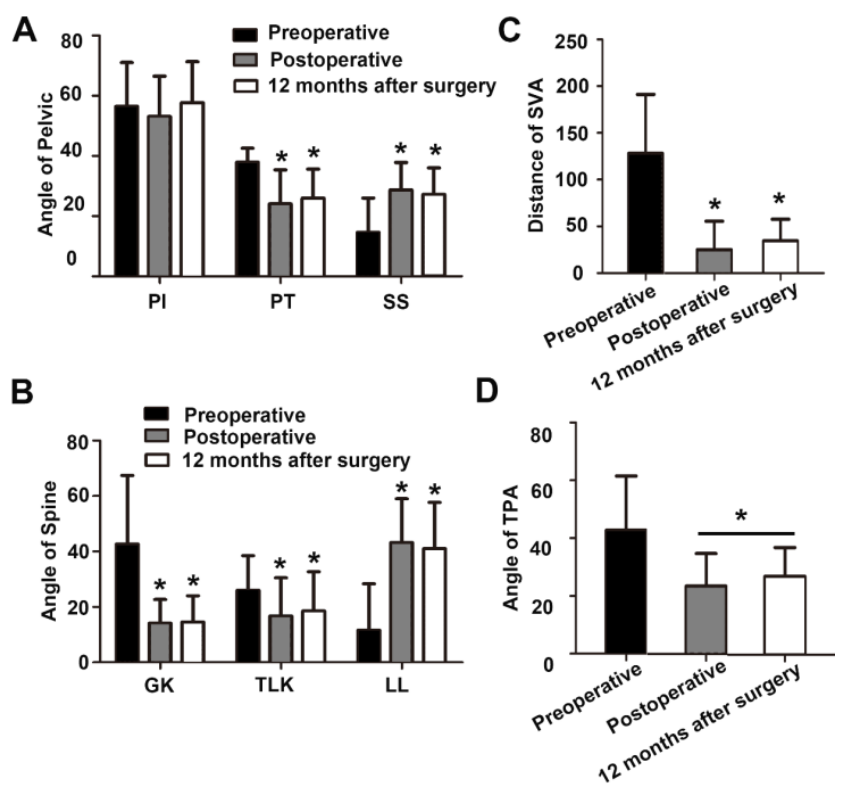

Figure 1. Spine-pelvic parameters were analyzed at pre-operation and postoperation.

A: Preoperative, postoperative and 12 months after surgery pelvic parameters comparison; B: Preoperative, postoperative and 12 months after surgery spinal parameters comparison; C: Preoperative, postoperative and 12 months after surgery SVA comparison; D: Preoperative, postoperative and 12 months after surgery TPA comparison (* means $P<0.05$ )

\subsection{ODI Were Analyzed at Pre-operation and 12 Months After Surgery}

Wilcoxon signed rank test using paired design Twosample comparison analysis of ODI scores before and 12 months after surgery, preoperative average walking ODI score of $3.00 \pm 0.82$ points, follow-up 12 months after 
follow-up $1.44 \pm 0.51$ The scores were statistically significant $(\mathrm{P}<0.05)$. The preoperative average ODI score was $2.63 \pm 0.72$ points, and the follow-up time was $1.44 \pm 0.51$ points after 12 months. The difference was statistically significant $(\mathrm{P}<0.05)$. The preoperative average ODI score was $2.87 \pm 0.89$, and the follow-up time was $1.56 \pm 0.73$ after 12 months. The difference was statistically significant $(\mathrm{P}<0.05)$. The mean total ODI score was $51.50 \pm 11.12$ points before operation, and $29.18 \pm 4.87$ points were followed up after 12 months. The difference was statistically significant $(\mathrm{P}<0.05)$. The scores of ODI were significantly improved at 12 months follow-up $(\mathrm{P}<0.05)$, and the patients were significantly improved in walking, sitting and standing.
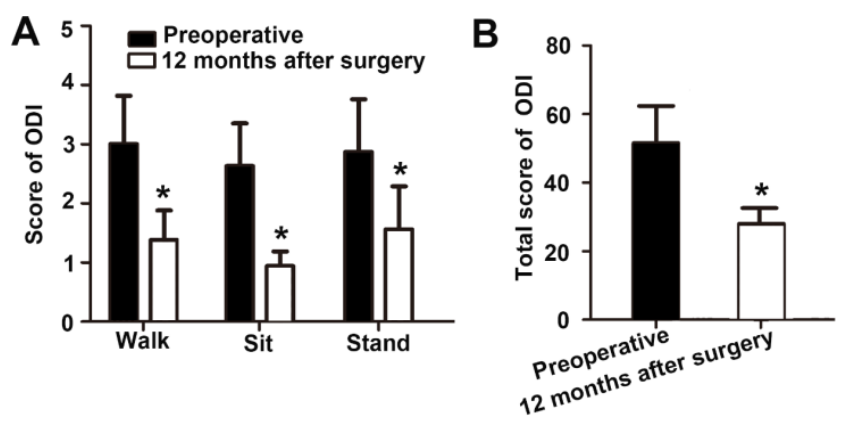

Figure 2. ODI were analyzed at pre-operation and 12 months after surgery.

A: Preoperative, 12 months after surgery ODI of Walk, Sit, stand comparison; B: Preoperative, 12 months after surgery Total score of ODI comparison. (* means $P<0.05$ )

\subsection{The Comparison of SRS-22 Score Between Preoperative and 12 Months After Surgery}

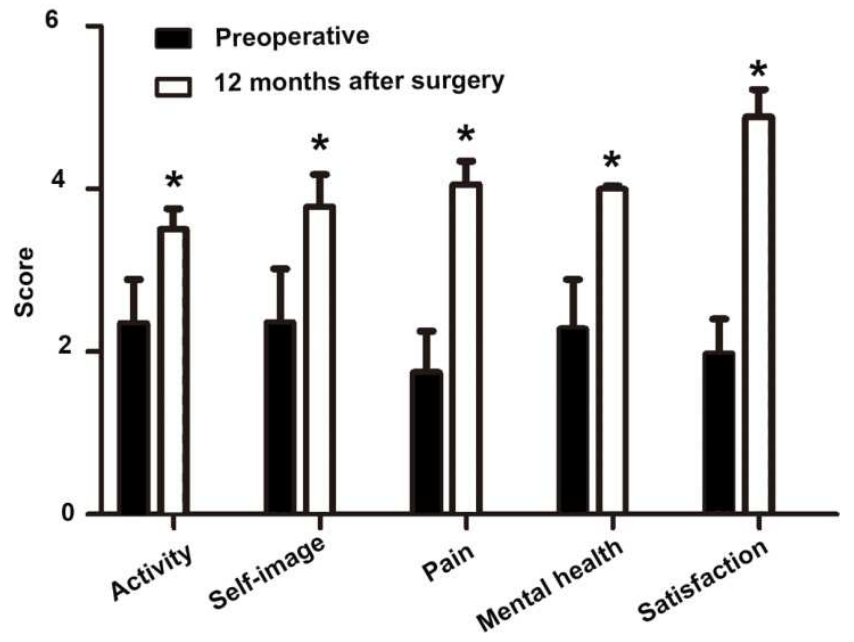

Figure 3. The comparison of SRS-22 score between Preoperative and 12 months after surgery.

Wilcoxon signed rank test using paired design two-sample comparison SRS-22 scores before and 12 months after surgery, SRS-22 score of $2.35 \pm 0.54$ before surgery, 12 months after surgery At follow-up of $3.50 \pm 0.26$, the difference was statistically significant $(\mathrm{P}<0.05)$. The average pain score of SRS-22 was $2.56 \pm 0.54$ points before operation, and $3.78 \pm 0.40$ points were followed up after 12 months. The difference was statistically significant $(\mathrm{P}<0.05)$. The preoperative average self-image SRS-22 score was $2.87 \pm 0.89$ points, and the follow-up time was $1.56 \pm 0.73$ points after 12 months. The difference was statistically significant $(\mathrm{P}<0.05)$. The preoperative average mental health item SRS-22 score was $2.28 \pm 0.61$ points, and the follow-up time was $3.99 \pm 0.05$ points after 12 months. The difference was statistically significant $(\mathrm{P}<0.05)$. The preoperative average satisfaction score of SRS-22 was $1.97 \pm 0.43$, and the follow-up was $4.88 \pm 0.34$ after 12 months. The difference was statistically significant $(\mathrm{P}<0.05)$.

\section{Discussion}

In patients with advanced spinal posterior kyphosis, the body weight of the AS will move forward, and the sagittal plane imbalance will occur. At this time, the body will compensate for the imbalance of the sagittal plane through the posterior pelvis and be in a hip extension, kneeling position. The hilar approach guides the osteotomy and orthopedic surgery to consider the post-compensation state of the pelvis, and more accurately guides the design of the osteotomy angle. According to the formula $\mathrm{tPT}=0.37 \times \mathrm{PI}-7^{\circ}$, the individualized kyphosis patients are personalized [6]. The orthopedic program avoids the patient's jaw angle $<0^{\circ}$, the maxillofacial view, restores the patient's head-up function and ensures the patient's standing, sitting and walking functions $[7,8]$. Common sagittal parameters of the pelvis include PI, PT, and SS. The pelvic incident angle PI was first proposed by Legaye is regarded as the anatomical parameter of the human pelvis [9]. It does not change with the change of body position. Different individuals have differences, but the measured values are relatively constant in the same adult individual. Ferguson et al proposed the sacral tilt angle SS, SS is the body position related parameters, the receptor position factor, and it has a significant correlation with the local parameters of lumbar vertebrae anterior horn LL [10]. The pelvic tilt angle PT also belongs to the pelvic body position correlation parameter, which is an important indicator reflecting the anterior and posterior tilt angle of the pelvis. Lafage found that PT can reflect the degree of compensation for spinal deformity by studying some patients with adult spinal deformity [11]. Most patients with AS kyphosis have compensated for sagittal imbalance by pelvic posterior tilt, hip hyperextension, and knee flexion (increased PT). Therefore, the correction of spinal deformity should also consider the posterior tilt state of the pelvis. Schwab et al [12] analyzed 70 patients with PSO osteotomy and surgery, found that in addition to PI, PT, SS were significantly improved than before surgery. The sagittal balance distance SVA has long been used to assess the sagittal balance of the spine of patients with AS kyphosis [13]. SVA reflects the severity of the sagittal deviation of the body in patients with AS kyphosis. As the SVA increases, the spine shifts more forward. It is very important to restore the sagittal balance for better functional outcomes [14]. For patients with AS kyphosis, in order to maintain the standing and walking 
function, it is necessary to strengthen the muscle strength of the lower back, which causes the back and back muscles of the patient to be more prone to fatigue and pain. Glassman et al proposed that the lumbar lordosis angle LL is an important local parameter affecting the quality of life of adult spinal deformity [15]. Christopher et al concluded that LL had a significant correlation with the VAS score of low back pain by analyzing 57 patients with spinal deformity [16]. Therefore, it is also necessary to restore good LL and SVA for osteotomy and orthopedic surgery in patients with AS kyphosis. Kim, Chang et al used PSO osteotomy to treat patients with AS kyphosis, and found that patients with LL and SVA were significantly improved [17]. Recently, some scholars have proposed TPA to reflect the sagittal balance of the spine-pelvis. TPA integrates spinal balance and pelvic compensatory effects to better guide treatment [18]. In this study, the spine-pelvis parameters PT, SS, GK, TLK, LL, SVA, and TPA were significantly improved after lung osteotomy guided osteotomy surgery. The quality of life of patients undergoing osteotomy and orthopedic surgery is also an important indicator for the evaluation of surgery. In this study, patients with postoperative osteotomy and orthopedic surgery have increased SRS-22 scores and decreased ODI dysfunction scores, indicating osteotomy orthopedics. After the patient's quality of life is significantly improved.

Spinal surgeons must carefully evaluate the spine-pelvic sagittal parameters and status of each AS kyphosis patient, and develop a personalized orthopedic plan based on the preoperative spine-pelvic sagittal parameters to achieve satisfactory postoperative outcomes. Sagittal orthopedic effect.

\section{Conclusion}

A good balance of the spine-pelvic sagittal sequence is necessary to ensure that the patient's normal walking, standing and other life tasks are maintained. The spine pelvis supports the sagittal balance of the torso of the patient. This study used multiple spinal parameters and pelvic parameters to evaluate the efficacy of the Hilus pulmonis to guide kyphosis correction. It was found that good image parameters were obtained after After using the Hilus pulmonis to guide kyphosis correction. Moreover, the postoperative SRS-22 function, self-image, and mental health scores were well scored before surgery, which basically satisfied the functions of head-up, standing, sitting, and lying in patients after orthopedic surgery. Therefore, the Hilus pulmonis to guide kyphosis correction for the treatment of AS kyphosis is worth promoting.

\section{Acknowledgements}

The authors declare no conflict of interest. This work was supported Natural Science Foundation of Guangdong Province, China (2017A030313595), Project of Educational Commission of Guangdong Province of China (2018KQNCX013), Medical Scientific Research Foundation of Guangdong Province of China (A2017184), The Fundamental Research Funds for the Central Universities
Project (21618304 and 21617498) and funded by China Postdoctoral Science Foundation (2019M653292).

\section{References}

[1] Braun J, Sieper J. Ankylosing spondylitis [J]. Lancet. 2007, 369 (9570): 1369-1390.

[2] Webers. C, Vanhoof L, Leue C. Depression in ankylosing spondylitis and the role of disease-related and contextual factors: a cross-sectional study. [J]. Arthrirtis Res Ther 2019, (21) 10: 215.

[3] Fei H, Li W S, Sun Z R, et al. Effect of patient position on the lordosis and scoliosis of patients with degenerative lumbar scoliosis [J]. Medicine, 2017, 96 (32): e7648.

[4] Debarge R, Demey G, Roussouly P. Sagittal balance analysis after pedicle subtraction osteotomy in ankylosing spondylitis [J]. European Spine Journal. 2011, 20 (5 Supplement): 619-625.

[5] Song K, Zheng G, Zhang Y, et al. Hilus pulmonis as the center of gravity for AS thoracolumbar kyphosis [J]. Eur Spine J. 2014, 23 (12): 2743-2750.

[6] Vialle R, Levassor N, Rillardon L, et al. Radiographic analysis of the sagittal alignment and balance of the spine in asymptomatic subjects [J]. J Bone Joint Surg Am. 2005, 87 (2): 260-267.

[7] Song K, Zheng G, Zhang Y, et al. A new method for calculating the exact angle required for spinal osteotomy [J]. Spine, 2013, 38 (10): E616-620.

[8] 2013, 38 (10): E616-620. Debarge R, Demey G, Roussouly P. Radiological analysis of ankylosing spondylitis patients with severe kyphosis before and after pedicle subtraction osteotomy [J]. Eur Spine J, 2010, 19 (1): 65-70.

[9] Legaye J, Duval-Beaupère G, Hecquet J, et al. Pelvic incidence: a fundamental pelvic parameter for threedimensional regulation of spinal sagittal curves $[\mathrm{J}]$. European Spine Journal. 1998, 7 (2): 99-103.

[10] Hu J, Ji ML, Qian BP, et al. Can pelvic tilt be predicated by the sacrofemoral-Pubic angel in patients with thoracolumbar kyphosis secondary to ankylosing spondylitis [J]. Spine. 2014, 39 (23): E1347-1352.

[11] Lafage V. Pelvic tilt and truncal inclination: two key radiographic parameters in the setting of adults with spinal deformity [J]. Spine (Phila Pa 1976). 2009, 34 (17): E599-606.

[12] Lafage V, Schwab F, Vira S, et al. Does vertebral level of pedicle subtraction osteotomy correlate with degree of spinopelvic parameter correction [J]. J Neurosurg Spine. 2011, 14 (2): 184-191.

[13] Qian BP, Jiang J, Qiu Y, et al. Radiographical predictors for postoperative sagittal Imbalance in patients With thoracolumbar kyphosis secondary to ankylosing spondylitis after lumbar pedicle subtraction osteotomy [J]. Spine. 2013, 38 (26): E1669-1675.

[14] Simon J, Longis PM, Passuti N. Correlation between radiographic parameters and functional scores in degenerative lumbar andthoracolumbar scoliosis [J]. Orthopaedics \& Traumatology. 2017, 2 (4): 182-187. 
[15] Glassman SD, Bridwell K, Dimar JR, et al. The impact of positive sagittal balance in adult spinal deformity [J]. Spine. 2004, 4 (5): S113-114.

[16] Christopher J. Sorensen, MS, et al. Is lumbar lordosis related to low back pain development during nrolonged standing? [J]. Man Ther. 2015, 20 (4): 553-557.

[17] Chang KW, Chen YY, Lin CC, et al. Closing wedge osteotomy versus opening wedge osteotomy in ankylosing spondylitis with thoracolumbar kyphotic deformity [J]. Spine. 2005, 30 (14): 1584-1593.

[18] Ryan DJ, Protopsaltis TS, Ames CP, et al. T1 pelvic angle (TPA) effectively evaluates sagittal deformity and assesses radiographical surgical outcomes longitudinally $[\mathrm{J}]$. Spine. 2014, 39 (15): 1203-1210 